\title{
Avancement des connaissances et capacité croissante pour faire face aux maladies infectieuses liées à la variabilité climatique au Canada
}

\author{
C Lee-Fuller'1, A Magnan'1, S Pharand ${ }^{1}$
}

\section{Résumé}

Le Cadre pancanadien sur la croissance propre et les changements climatiques a été adopté en décembre 2016. Ce rapport mis au point de manière collaborative avec les gouvernements fédéral, provinciaux et territoriaux documente les plans canadiens qui visent à respecter les engagements que le Canada a pris à travers l'Accord de Paris et à stimuler l'économie canadienne. Le rapport relève une gamme de mesures regroupées au sein de quatre piliers : la tarification du carbone, les mesures complémentaires visant à réduire les émissions, l'adaptation et la résilience aux changements climatiques et le soutien à la croissance économique grâce aux technologies propres, à l'innovation et à l'emploi. La protection et l'amélioration de la santé et du bien-être, qui sont des aspects essentiels de l'adaptation et de la résilience climatique, ont également été inclus dans le Cadre pancanadien. De nouvelles mesures liées au Cadre pancanadien comprenaient des mesures fédérales d'envergure visant à la prévention des maladies liées à la canicule (un dossier pris en charge par Santé Canada), et à la réduction des risques associés aux maladies infectieuses liées à la variabilité climatique (un dossier pris en charge par l'Agence de la santé publique du Canada [subséquemment identifié comme l'Agence]).

Les spécialités de la santé publique et des changements climatiques se recoupent en ce qui a trait au domaine des maladies infectieuses. Afin de mener ses nouveaux engagements à bonne fin en ce qui a trait au Cadre pancanadien, I'Agence a élaboré le programme de maladies infectieuses et de changements climatiques (PMICC) et a institué un nouveau Fonds comptant des subventions et des contributions pour soutenir le programme. Le programme comporte trois volets principaux : augmenter la capacité de l'Agence à répondre au nombre croissant de demandes face aux maladies infectieuses liées à la variabilité climatique; fournir aux Canadiens l'accès à des renseignements opportuns leur permettant de mieux comprendre leurs risques et de mieux se protéger contre les infections; augmenter l'adaptabilité ou la résilience aux impacts des maladies infectieuses grâce à la surveillance et au contrôle, à l'amélioration des capacités diagnostiques en laboratoire et à l'accès à des outils de sensibilisation et d'éducation. Au cours de sa première année, plusieurs projets de dépistage et de surveillance ainsi qu'en matière de sensibilisation et d'éducation ont été subventionnés par le fonds du PMICC. En collaboration avec les acteurs clé et les gouvernements de tous les niveaux dans l'ensemble des provinces et des territoires, l'Agence continuera d'œuvrer à accroître la sensibilisation aux effets des changements climatiques sur la prévalence des maladies infectieuses et d'aider les Canadiens à se préparer aux impacts anticipés et non anticipés.
Citation proposée : Lee-Fuller C, Magnan A, Pharand S. Avancement des connaissances et capacité croissante pour faire face aux maladies infectieuses liées à la variabilité climatique au Canada. Relevé des maladies transmissibles au Canada 2018;44(10):303-6. https://doi.org/10.14745/ccdr.v44i10a06f
Affiliation

${ }^{1}$ Centre des maladies infectieuses d'origine alimentaire, environnementale et zoonotique, Agence de la santé publique du Canada, Ottawa (Ontario)

*Correspondance: phac. cfezid-cmioaez.aspc@canada.ca

Mots clés : Changements climatiques, maladies infectieuses, résilience, adaptation, surveillance, éducation, sensibilisation, capacité 


\section{Introduction}

Reconnaissant la nécessité d'agir pour contrer les changements climatiques, les premiers ministres du Canada ont adopté le Cadre pancanadien sur la croissance propre et les changements climatiques en décembre 2016 (1). Ce rapport mis au point de manière collaborative avec les gouvernements fédéral, provinciaux et territoriaux documente les plans canadiens qui visent à respecter les engagements que le Canada a pris à travers l'Accord de Paris (2) et à stimuler l'économie canadienne. Les gouvernements provinciaux et territoriaux qui appuient le Cadre pancanadien et le gouvernement fédéral ont identifié une gamme de mesures regroupées au sein de quatre piliers : la tarification du carbone, les mesures complémentaires visant à réduire les émissions, l'adaptation aux changements climatiques et la capacité de leur résister, et le soutien à la croissance économique grâce aux technologies propres, à l'innovation et à l'emploi.

Le Cadre pancanadien reconnaît qu'il appartient à tous de se responsabiliser vis-à-vis les changements climatiques et que chacun - tous les niveaux de gouvernement, les organisations autochtones, les collectivités, I'industrie, les organisations non gouvernementales et les citoyens à l'échelle du pays a un rôle à jouer. Le Cadre pancanadien comprend plus de cinquante mesures concrètes à mettre en œuvre par rapport aux changements climatiques, tant dans toutes les provinces et tous les territoires que dans tous les secteurs. De plus, il appuie également la mise en œuvre des Objectifs de développement durable des Nations Unies 2030 (3).

La protection et l'amélioration de la santé et du bien-être, lesquels sont des éléments essentiels de l'adaptation et de la résilience climatique, ont également été inclus dans le Cadre pancanadien sur la croissance propre et les changements climatiques. D'autres domaines d'action prioritaire de ce pilier comprenaient la communication des renseignements scientifiques et de connaissances traditionnelles en mesures pratiques, l'augmentation de la résilience climatique au moyen de l'infrastructure, le soutien aux régions particulièrement vulnérables et la réduction des risques liés au climat et aux catastrophes. L'inclusion de la santé et du bien-être, en tant que composants clés du Cadre pancanadien, a permis de reconnaître le fait que le fardeau et l'impact sur la santé des Canadiens vont augmenter à mesure que les changements climatiques progresseront. Malheureusement, les populations vulnérables et à risque seront les premières à subir les conséquences des changements climatiques. Pour cette raison, de nouvelles mesures dans le cadre du plan comprennent des mesures fédérales de plus grande envergure qui permettront d'éviter les maladies causées par les canicules, de réduire les risques de maladies infectieuses liées à la variabilité climatique et de soutenir les communautés autochtones et des Nations à poursuivre des activités en matière de santé.
On a également responsabilisé Santé Canada, l'Agence, les Instituts de recherche en santé du Canada et ses partenaires en comptant sur eux pour faire progresser la science, les connaissances et les pratiques exemplaires, et ainsi faciliter I'adaptation aux changements climatiques. L'Agence possède une bonne expérience en matière de programmes dans ce secteur; elle fait figure de chef de file en santé publique pour ce qui est des répercussions des changements climatiques sur les maladies infectieuses en général.

L'objectif du présent article est de souligner brièvement le rôle de la santé publique dans l'adaptation aux changements climatiques et de décrire le nouveau programme de maladies infectieuses et de changements climatiques (PMICC) que l'Agence a lancé, incluant un nouveau programme de financement.

\section{La santé publique et les changements climatiques}

Les programmes de santé publique jouent un rôle important dans la sensibilisation aux effets des changements climatiques en dotant le grand public, les professionnels de la santé et les décideurs à différents niveaux des gouvernements d'outils et d'informations afin d'aider la population canadienne à se préparer aux impacts des changements climatiques et à augmenter sa résilience. Le rôle des organismes de santé publique en matière de changements climatiques exige l'établissement de nouveaux partenariats, et des collaborations inédites grâce à des initiatives multi-juridictionnelles et multidisciplinaires.

Les domaines de la santé publique et des changements climatiques des maladies infectieuses se recoupent. Un exemple des effets directs et indirects que les changements climatiques ont sur les maladies infectieuses et où nos connaissances continuent d'évoluer est le risque de maladies vectorielles. Les modifications de la répartition géographique, des habitats et du caractère saisonnier des maladies transmises par vecteur font en sorte que des maladies infectieuses relativement rares envahissent de nouvelles zones ou que de nouvelles maladies auparavant absentes au Canada émergent (4). Les récents investissements du gouvernement fédéral à l'Agence, tant dans le Centre des maladies infectieuses d'origine alimentaire, environnementale et zoonotique que dans le Laboratoire national de microbiologie qui mènent des recherches sous les auspices du Cadre pancanadien sur la croissance propre et les changements climatiques traduisent les efforts qui ont été axés sur l'accroissement de la capacité et de la compréhension des maladies infectieuses liées à la variabilité climatique, y compris les maladies transmises par vecteur, et ce, de manière à pouvoir les traiter, grâce à des processus de surveillance et de dépistage, d'évaluation des risques, de modélisation et des diagnostics de laboratoire, ainsi qu'à des activités de 
sensibilisation publique et de formation des professionnels de la santé (5). Ces investissements reflètent le constat selon lequel les conséquences des changements climatiques et d'une préparation inadéquate à ces changements seraient incommensurables.

\section{Programme de maladies infectieuses et de changements climatiques de l'Agence}

Afin de mener ses nouveaux engagements à bonne fin en ce qui a trait au Cadre pancanadien, I'Agence a élaboré le PMICC en 2016. Ce programme mise sur des programmes antérieurs, dans les domaines indiqués ci-dessus, et il contribuera également à faire progresser le travail réalisé par le Cadre fédéral sur la maladie de Lyme (6) et le processus de subvention du réseau de recherche sur la maladie de Lyme sous l'égide des Instituts de recherche en santé du Canada (7).

Le programme de l'Agence est surtout axé sur les maladies infectieuses liées à la variabilité climatique (y compris les maladies à transmission vectorielle), ainsi que sur les maladies infectieuses d'origine hydrique et d'origine alimentaire, et il comprend un nouveau fonds de subventions et de contributions. Le programme comporte trois volets principaux : augmenter la capacité de l'Agence à répondre au nombre croissant de demandes face aux maladies infectieuses liées à la variabilité climatique; fournir aux Canadiens l'accès à des renseignements opportuns leur permettant de mieux comprendre leurs risques et à mieux se protéger contre l'infection; augmenter l'adaptabilité ou la résilience aux impacts des maladies infectieuses grâce à la surveillance et au contrôle, à des capacités diagnostiques en laboratoire améliorées et à l'accès à des outils de sensibilisation et d'éducation.

En août 2017, l'Agence a lancé le Fonds du PMICC, une enveloppe de deux millions de dollars offerte annuellement sous forme de subventions et de contributions pour le financement de projets sur une période de 11 ans. Le Fonds sert de nouveau véhicule à l'Agence pour faire progresser son travail sur les maladies infectieuses liées à la variabilité climatique au Canada et lorsque c'est possible, le travail lié à l'approche Une seule santé. Le financement est alloué sur la base de sollicitations dirigées, ciblées et ouvertes. II comprend deux secteurs prioritaires :

- le contrôle et la surveillance, ainsi que

- la sensibilisation et l'éducation.

Les activités liées à la surveillance aideront à établir des données de référence et des mesures de contrôle qui permettront de mieux prédire les maladies infectieuses liées à la variabilité climatique et d'intervenir efficacement. Ce qui sera fait en analysant la propagation des maladies infectieuses (entre autres, virus, bactéries, parasites, champignons et maladies à prions), particulièrement dans les collectivités mal desservies.

Les activités d'éducation et de sensibilisation comprendront l'élaboration, l'adoption et la distribution de matériel destiné aux professionnels de la santé et la distribution d'outils et du savoir sur les pratiques exemplaires dans toutes les collectivités canadiennes, y compris les populations vulnérables.

Les projets pour lesquels le financement a été accordé dans le cadre du PMICC incluent des organismes professionnels en santé, des universités, des communautés autochtones et des provinces qui font progresser les connaissances sur les répercussions des maladies infectieuses liées à la variabilité climatique sur la santé humaine ont pu s'en prévaloir. Ces projets financés par l'Agence permettront d'améliorer les connaissances de base grâce à la surveillance sur le terrain des populations de tiques, à des études sur les risques liés aux maladies infectieuses dans des régions spécifiques au Canada et à l'élaboration de nouveaux outils, de formations et de ressources destinés aux professionnels de la santé ainsi qu'aux populations et aux communautés vulnérables. Nous attendons l'annonce du financement des projets.

L'Agence prépare actuellement le prochain processus de sollicitation de fonds dans le cadre du PMICC — prévu pour l'automne 2018 - pour des projets débutant en 2019-2020 et pour les prochaines années du programme. Des renseignements détaillés sur les cibles de cette sollicitation seront accessibles sur le site Web canada.ca et par courriel à travers les responsables du programme.

\section{Conclusion}

Les répercussions des changements climatiques sont de plus en plus visibles dans le monde entier. Au Canada, les gouvernements provinciaux et territoriaux qui appuient le Cadre pancanadien et le gouvernement fédéral ont précisé les mesures essentielles pour contrer ces changements. Nous reconnaissons la nécessité de nous concentrer sur la santé et le bien-être dans le cadre de nos mesures d'adaptation.

L'Agence s'engage à s'attaquer aux répercussions des changements climatiques sur les maladies infectieuses, et a relevé les lacunes sur les plans des connaissances et de la capacité qui doivent être comblées afin de mieux contrer I'incidence croissante actuelle et future des maladies infectieuses liées à la variabilité climatique. L'Agence continue d'augmenter ses connaissances et son expertise afin de soutenir la mise en $œ u v r e$ du Cadre pancanadien. À preuve, la mise en place par I'Agence du nouveau PMICC qui s'accompagne d'un fonds de subventions et de contributions.

L'Agence continuera à œuvrer pour faire progresser la sensibilisation aux effets des changements climatiques et 
pour accroître les connaissances en la matière tout en aidant les Canadiens à se préparer aux répercussions et à y résister.

\section{Références}

1. Cadre pancanadian sur la croissance propre et les changements climatiques: plan canadien de lutte contre les changements climatiques et de croissance économique. Ottawa (ON): Gouvernement du Canada; 2016. https://www. canada.ca/content/dam/themes/environment/documents/ weather1/20170125-fr.pdf

2. The Paris Agreement. 2015. https://unfccc.int/process-andmeetings/the-paris-agreement/the-paris-agreement

3. UN 2030 Sustainable Development Goals. https:// sustainabledevelopment.un.org/sdgs

4. Ogden NH. Climate change and vector-borne diseases of public health significance. FEMS Microbiol Lett 2017
Oct;364(19). https://doi.org/10.1093/femsle/fnx186. PubMed (https://www.ncbi.nlm.nih.gov/pubmed/28957457)

5. Premier rapport annuel du Cadre pancanadien sur la croissance propre et les changements climatiques : résumé. Ottawa (ON); Governement du Canada: 2017. https://www.canada.ca/fr/services/environnement/meteo/ changementsclimatiques/cadre-pancanadien/premierrapport-annuel/resume.html

6. La maladie de lyme au Canada-Cadre fédérale. Agence de la santé publique du Canada. Ottawa (ON); ASPC; 2017. https://www.canada.ca/fr/sante-publique/services/ publications/maladies-et-affections/maladie-lyme-canadacadre-federal.html

7. Pre-announcement: Lyme Disease Research Network. Canadian Institutes of Health Research. ClHR; 2017. www. cihr-irsc.gc.ca/e/50552.html 